\title{
DIFERENÇAS CONCEITUAIS E EMPÍRICAS ENTRE EFICÁCIA ADAPTATIVA E COPING
}

Tales Vilela Santeiro

Universidade Federal do Triangulo Mineiro - UFTM

Elisa Medici Pizão Yoshida

Pontifícia Universidade Católica de Campinas - PUCC

Evandro Morais Peixoto

Pontifícia Universidade Católica de Campinas - PUCC

Glaucia Mitsuko Ataka da Rocha

Universidade de São Paulo - USP

Daniela Sacramento Zanini

Pontifícia Universidade Católica de Goiás - PUCG

\section{Resumo}

Nessa pesquisa, medidas da eficácia adaptativa e de coping são comparadas, com o objetivo de se analisar em que medida elas refletem aspectos semelhantes do funcionamento geral das pessoas. A amostra foi composta por 103 estudantes universitários, com idades entre 17 e 44 anos (21,67 $\pm 4,78 ; 83,5 \%$ mulheres). A Escala Diagnóstica Adaptativa Operacionalizada de Autorrelato (EDAO-AR) e o Coping Response Inventory - Adult Form (CRI-A) foram instrumentos utilizados. Os escores foram analisados por meio testes- $t$ para amostras independentes e correlação de Person. Apenas para a estratégia de coping "Descarga emocional" pontuações significativamente maiores para as mulheres foram observadas $(t(97)=0,930, p=0,02$, $d=0,2)$. Apesar de a EDAO-AR e CRI-A guardarem entre si graus de associação e de sobreposição teórica, os resultados indicaram que os construtos eficácia adaptativa e coping focalizam aspectos diferentes das atividades humanas. Limites da pesquisa e perspectivas futuras são discutidos.

Palavras-chave: escalas; avaliação psicológica; enfrentamento; universitários; jovens.

\section{CONCEPTUAL AND EMPIRICAL DIFFERENCES BETWEEN ADAPTIVE EFFICACY AND COPING}

\begin{abstract}
In this research, measures of adaptive efficacy and coping are compared with the aim of examining the extent to which they reflect similar aspects of the general functioning of individuals. The sample consisted of 103 undergraduate students, aged between 17 and 44 years $(21.67 \pm 4.78 ; 83.5 \%$ women $)$. The instruments were The Escala Diagnóstica Adaptativa Operacionalizada de Autorrelato (EDAO-AR) and the Coping Response Inventory - Adult Form (CRI-A). We used $t$-test for independent samples and Pearson correlation for data analysis. Significant higher scores for women were observed only for coping strategy "emotional discharge" $(t(97)=0.930, p=0.2, d=$ $0.2)$. Although the EDAO-AR and the CRI-A keep with each other some degree of association and theoretical overlap, the results indicated that the constructs of adaptive efficacy and coping focuses on different aspects of human activities. Limitations of the research and new perspectives are discussed.
\end{abstract}

Keywords: Scaling (testing); psychological assessment; coping behavior; college students; young adults. 


\title{
DIFERENCIAS CONCEPTUALES Y EMPÍRICAS ENTRE EFICACIA ADAPTATIVO Y AFRONTAMIENTO
}

\begin{abstract}
Resumen
En esta investigación, las medidas de eficiencia de adaptación y afrontamiento se comparan con el fin de analizar en qué medida reflejan aspectos similares del funcionamiento general de las personas. La muestra estuvo constituida por 103 estudiantes universitarios, de edades comprendidas entre los 17 y 44 años $(21,67 \pm$ 4,$78 ; 83,5 \%$ mujeres). La escala Diagnostica Adaptativa Operacionalizada de Autorrelato (EDAO-AR) y el Coping Response Inventory - Adult Form (CRI-A) fueron los instrumentos utilizados. Los resultados fueron analizados pruebas $t$ para muestras independientes y correlación de Pearson. Sólo para la estrategia de afrontamiento "Descarga Emocional" se observaran puntuaciones significativamente más altas para las mujeres ( $t(97)=0,930, p=0,02, d=0,2$ ). A pesar de la EDAO-AR-A y el CRI presentaren grados de asociación y de superposición teórica, los resultados indicaron que los constructos de eficacia adaptativa y afrontamiento se centran en diferentes aspectos de la actividad humana. Se discuten las limitaciones de la investigación y perspectivas de futuro.
\end{abstract}

Palabras clave: escalas; evaluación psicológica; enfrentamiento; estudiantes universitarios; jóvenes.

\section{INTRODUÇÃO}

No estágio atual da psicologia, em que múltiplos enfoques teóricos coexistem, surge com frequência a impressão de que diferentes conceitos se referem a um mesmo fenômeno. Ou seja, fala-se do mesmo por meio de termos diferentes. Trata-se apenas de uma questão semântica, ou haveria algum tipo de relação entre eles? Outra questão que se coloca é saber se conceitos provenientes de modelos teóricos diferentes podem eventualmente ser utilizados de forma complementar na compreensão de uma condição ou fenômeno humanos (Norcross, 2011). Nas últimas décadas, temos nos dedicado ao estudo da eficácia adaptativa, constructo proposto por Simon (1983/1989) e, mais recentemente, ao de coping (Folkman \& Lazarus, 1980), com aproximações teóricas distintas, mas que podem guardar certa afinidade em suas operacionalizações. De acordo com Schinke, Tenenbaum, Lidor e Battochio (2010) e Schinke et al. (2012) a adaptação pode ser entendida como um processo, que começa com a avaliação de um estressor, passa pelo emprego de estratégias efetivas e termina com uma resposta adaptativa propriamente dita, que pode ser adequada ou não, a depender das características das etapas anteriores. Nesta direção, o tipo de estratégia empregada passa a ter um papel crucial no tipo de respostas adaptativas a serem emitidas pelos sujeitos. Por conseguinte, nessa pesquisa, avaliações da eficácia adaptativa e de coping são comparadas e discutidas as aproximações conceituais e empíricas.

No que diz respeito ao conceito de adaptação e respectiva medida, Simon (1983/1989), partindo de uma perspectiva clínica preventiva, ocupou-se da descrição do processo de evolução adaptativa, tendo como objetivo "a prevenção da perda da eficiência da adaptação" (p.11). Definiu adaptação como o "conjunto de respostas de um organismo vivo, em vários momentos, a situações que o 
modificam" (p.14). O propósito é o de responder às situações de vida, no sentido de solucionar as situações-problema e manter a integração do organismo (Simon, 1997). No entanto, o autor considerou que "a adaptação, em si mesma só permite separar os organismos vivos dos mortos" (p. 15) e propôs avaliar o tipo de resposta que o sujeito apresenta para a solução de seus problemas, para a satisfação de suas necessidades. O modo com que o organismo consegue responder às situações e contingências de sua vida traduziria, portanto, sua eficácia adaptativa, a qual reflete a integração dos vários sistemas, internos (psíquicos) e externos (socioculturais) da pessoa, bem como a coerência de suas ações.

A avaliação da eficácia adaptativa é realizada por meio da Escala Diagnóstica Adaptativa Operacionalizada/EDAO (Simon, 1983/1989). Na versão original da escala, a avaliação é feita com base em raciocínio clínico, a partir de uma entrevista com a pessoa. Avalia-se a adequação das respostas dadas para a solução dos problemas em quatro setores da personalidade - Afetivo-Relacional (A-R), Produtividade (Pr), Sócio-Cultural (S-C) e Orgânico (Or) (Simon, 1983/1989). Sendo que os dois primeiros, considerados mais relevantes para a configuração da adaptação, são avaliados quantitativamente e os setores S-C e Or são avaliados qualitativamente (Simon, 1997, 2005).

Para determinar a adequação das respostas em cada setor, elas são avaliadas como adequadas, pouco adequadas ou pouquíssimo adequadas. As primeiras consideram haver uma resposta para solução do problema, que traga satisfação ao indivíduo e não provoque conflitos intrapsíquicos (coerência da solução com valores internos), nem socioculturais. As pouco adequadas não satisfazem a um dos dois últimos critérios: não trazem satisfação ou geram algum tipo de conflito, interno ou externo. Já as pouquíssimas adequadas apenas dão uma resposta ao problema em questão, no entanto, não trazem satisfação e ainda geram algum tipo de conflito (Simon, 1983/1989, 1997, 2005). Do conjunto das respostas dadas nos setores A-R e Pr chega-se à configuração geral da eficácia adaptativa expressa pelos seguintes grupos classificatórios (melhor detalhados no item Instrumentos): Gr.1 Adaptação Eficaz, Gr.2 Adaptação Ineficaz Leve, Gr.3 Adaptação Ineficaz Moderada, Gr. 4 Adaptação Ineficaz Severa, Gr. 5 Adaptação Ineficaz Grave (Simon, 1997; 2005).

Para situações em que entrevistas clínicas não são viáveis, foi idealizada uma versão de autorrelato da EDAO (EDAO-AR) (Yoshida, 2012), composta por uma escala que avalia a adequação das respostas do setor A-R e outra que mede a adequação das respostas do setor da Pr. É possível classificar o sujeito quanto à eficácia adaptativa pela somatória dos escores médios obtidos nas duas escalas e chegar ao grupo de classificação da eficácia adaptativa, nos mesmos termos que na versão clínica de Simon (1997). Quanto às qualidades psicométricas da EDAO-AR, o estudo de Yoshida (2013) demostrou que a estrutura fatorial de cada subescala foi constituída por três dimensões: foco na situação problema, 
foco na relação interpessoal e foco no eu, com índices de consistência interna muito bons (alfa de Cronbach $\geq 0,80$ ) para a escala geral e para as duas escalas ( $A-R$ e Pr). Além disso, evidências de validade baseadas na relação com outras variáveis demonstraram associações negativas entre a EDAO-AR e a severidade de sintomas psicopatológicos, medidos com a Escala de Avaliação de Sintomas40 (EAS-40) (Laloni, 2001)

Retomando a questão do conceito de eficácia da adaptação, brevemente descrito acima (mais detalhes podem ser obtidos nos trabalhos de Simon, 1997, 2005, ou ainda na revisão de Yoshida, Enéas, \& Santeiro, 2010), é possível dizer que o conceito de eficácia adaptativa permite qualificar a forma como a pessoa vem enfrentando as vicissitudes da vida (Simon, 1983/1989). Ou seja, diz respeito à qualidade das formas de enfrentamento e, por conseguinte, de quão bem vem conseguindo manter sua integridade psíquica. Essa noção de enfrentamento encontra-se também no âmago do conceito de coping, desenvolvido segundo o modelo relacional/cognitivo (Lazarus \& Folkman, 1984). Apesar de provirem de matrizes teóricas diversas, portanto, ambos parecem guardar algum tipo de relação ou de associação entre si.

A designação coping tem sido atribuída ao conjunto de esforços cognitivos e comportamentais que a pessoa utiliza para lidar com demandas específicas, internas ou externas, que surgem em situações estressoras e que são avaliadas como sobrecarga em relação aos recursos pessoais (Lazarus \& Folkman, 1984). Esta definição envolve quatro aspectos básicos a serem considerados: (1) coping é um processo ou uma interação que se dá entre o indivíduo e o ambiente; (2) a função do coping é de administração da situação estressora, ao invés de controle ou domínio da mesma; (3) os processos de coping pressupõem a noção de avaliação sobre como certo fenômeno é percebido, interpretado e representado pelo indivíduo; e (4) o processo de coping constitui-se em uma mobilização de esforços cognitivos e comportamentais para administrar (reduzir, minimizar ou tolerar) as demandas internas ou externas que surgem da sua interação com o ambiente (Folkman \& Lazarus, 1980).

Pode-se dizer que coping são as estratégias assumidas frente a um determinado evento estressor que pode ter como resultado a adaptação geral do indivíduo ou a manutenção de sua saúde mental ou, pelo contrário, adoecer o indivíduo. Além disso, as ações de coping são deliberadas e podem ser aprendidas ao longo da vida do indivíduo. Assim, as formas como se enfrenta um problema podem variar segundo a pessoa, seu sexo, idade, contexto social, o tipo de problema enfrentado, os recursos psicológicos e sociais de que dispõe para o enfrentamento, entre outros aspectos (Zanini, Forns, \& Kirchner, 2005).

Estudos sobre coping e sobre sua avaliação, por sua vez, têm sido observados em diversas realidades. Para além da norte-americana onde a conceituação foi idealizada e permanece sob enfoque (por exemplo, Folkman, 2011), especificamente na realidade brasileira e latinoamericana há evidências 
de que investigações sobre coping têm sido crescentes nos últimos 20 anos (Santeiro, Zanini, \& Santeiro, 2013).

Dentre os instrumentos desenvolvidos para avaliar coping, o Coping Response Inventory - Adult Form (CRI-A) (Moos, 1993) permite avaliar as respostas do indivíduo frente aos eventos estressores segundo dois grupos de estratégias: as de aproximação e as de evitação. As estratégias de aproximação são equivalentes às de coping focalizado no problema, descritas por Lazarus e Folkman (1984), e referem-se aos esforços cognitivos e comportamentais para domínio ou resolução dos estressores da vida. Este grupo de estratégias é formado pelas estratégias do tipo Análise lógica, Reavaliação positiva, Busca de apoio e Resolução do problema.

As estratégias do grupo de evitação, por sua vez, são equivalentes às de coping focalizado na emoção (Lazarus \& Folkman, 1984) e contemplam os esforços cognitivos e comportamentais para evitar o problema ou administrar os sentimentos negativos causados por ele. São elas: Evitação cognitiva, Aceitação/resignação, Busca de gratificação alternativa e Descarga emocional (a descrição mais detalhada de cada uma das estratégias específicas será realizada no apartado dos instrumentos).

São estratégias de Aproximação ao problema: Análise lógica - inclui tentativas cognitivas realizadas para compreender e preparar-se mentalmente para o enfrentamento da situação estressante e suas consequências; Reavaliação positiva - corresponde a tentativas cognitivas de construir e reestruturar a situação estressante de forma positiva; Busca de apoio - tentativas comportamentais de busca de informação, apoio e orientação; Resolução de problemas - tentativas comportamentais de ações diretas para solução do problema. São estratégias de Evitação: Evitação cognitiva - inclui tentativas cognitivas para evitar pensamentos relacionados ao problema; Aceitação/Resignação - tentativas cognitivas para aceitação do problema ou para adotar uma atitude de resignação ao mesmo; Busca de gratificação alternativa tentativas comportamentais para envolver-se em atividades substitutivas que gerem satisfação e evitem o mal-estar causado pela vivência do problema; e Descarga emocional - tentativas comportamentais de reduzir a tensão da vivência do problema por meio da expressão dos sentimentos negativos.

A versão do CRI-A empregada está sendo adaptada ao português com aprovação do autor (Richard Moos) e editora (Psychological Assessment Resources, Inc.) que o comercializa. O CRI-A foi traduzido e adaptado a diferentes culturas (ex.: Aguilar-Vafaie, \& Abiari, 2007; Kirchner, Forns, Muñoz, \& Pereda, 2008; Kohli, Batra, \& Aggarwal, 2011) apresentando qualidades psicométricas satisfatórias e condizentes com os instrumentos de avaliação de coping (Aldwin, 1994). No Brasil, este instrumento vem sendo utilizado com diferentes grupos amostrais a fim de verificar sua aplicabilidade. Os dados, apontados nas publicações, indicam qualidades psicométricas satisfatórias e 
estrutura fatorial similares à de estudos internacionais. Ademais, para os grupos de estratégias de coping de Aproximação e Evitação, os índices de consistência interna (alfa de Cronbach) são adequados, variando de 0,68 a 0,80 em diversos estudos com amostras de crianças, adolescentes, estudantes universitários e em pacientes com câncer (Paula Junior \& Zanini, 2011; Zanini, Mendonça, Forns, \& Kirchner, 2010; Zanini \& Queiroz, 2011). Por este motivo optou-se por usar este instrumento de medida neste estudo.

Com base nos conceitos de eficácia adaptativa e de coping apresentados, verifica-se que o primeiro diz respeito à qualidade do enfrentamento de situações que se apresentam ao indivíduo nos diferentes setores de funcionamento - A-R, $\mathrm{Pr}, \mathrm{S}-\mathrm{C}$ e Or -, enquanto que a noção de coping focaliza especialmente as estratégias utilizadas pelo indivíduo, no enfrentamento das situações estressantes e de sobrecarga, independentemente do setor da personalidade envolvido.

Em face à aparente superposição de aspectos implicados nos conceitos eficácia adaptativa e de coping, o presente estudo teve como objetivo investigar possíveis relações empíricas entre eles. Com este objetivo, foram obtidas medidas da eficácia adaptativa e de coping de universitários, medidos pela EDAO-AR e pela CRI-A. A expectativa é de que correlações positivas, mas moderadas, sejam encontradas entre a avaliação da eficácia adaptativa avaliada pela EDAO-AR e o conjunto de escalas que avaliam as estratégias de aproximação do CRI-A. Quanto às estratégias de evitação, avaliadas com o CRI$A$, são esperadas correlações negativas e moderadas com a EDAO-AR. Considerou-se ainda que se as correlações fossem muito altas, seria indicativo de que ambos os instrumentos estariam avaliando o mesmo construto.

Com base nos conceitos de eficácia adaptativa e de coping apresentados, verifica-se que o primeiro diz respeito à qualidade do enfrentamento de situações que se apresentam ao indivíduo nos diferentes setores de funcionamento - A-R, $\mathrm{Pr}$, S-C e Or -, no entanto, chegando a um conceito mais amplo de eficácia adaptativa. Já, a noção de coping focaliza especialmente as estratégias utilizadas pelo indivíduo, no enfrentamento das situações estressantes e de sobrecarga, independentemente do setor da personalidade envolvido. Há, portanto, uma aparente superposição de aspectos implicados nos dois conceitos. Por conseguinte, o presente estudo teve como objetivo verificar, empiricamente, se haveria associação entre os construtos eficácia adaptativa e coping.

\section{MÉTODO}

\section{Participantes}

A amostra, por conveniência, foi composta por 103 estudantes universitários, com idades que variaram entre 17 e 44 anos (M: 21,67 $\pm 4,78$; 
$83,5 \%$ mulheres). Sobre o estado civil, $94,2 \%$ eram solteiros, $4,8 \%$ casados e $1,0 \%$ divorciados. Quanto à origem dos participantes, esses eram matriculados no curso de Psicologia em instituição pública de ensino superior, situada na região Centro-Oeste do Brasil.

\section{Instrumentos}

\section{Escala Diagnóstica Adaptativa Operacionalizada de Autorrelato (EDAO-AR)} (Yoshida, 2012)

Instrumento desenvolvido para avaliar a eficácia adaptativa, de acordo com duas escalas: Afetivo-Relacional $(A-R)$ e Produtividade ( $P r)$. A escala A-R afere ações, sentimentos e atitudes do indivíduo em relação a outros significantes e em relação a si mesmo. A escala da Pr avalia ações, sentimentos e atitudes do indivíduo, relacionados a qualquer atividade produtiva considerada principal no momento da aplicação da escala (Simon, 1997). Utilizou-se a segunda versão da Escala, com 45 itens, dos quais 24 avaliam a qualidade da adaptação do setor A-R e 21 do setor da Pr. Para cada item há três alternativas de respostas correspondentes aos níveis de qualidade adaptativa, ponderadas de acordo com os critérios propostos por Simon (1997): adaptação adequada, pouco-adequada e pouquíssimo-adequada. No setor A-R os itens são pontuados respectivamente com os escores, 3, 2 e 1; e no setor $\operatorname{Pr}, 2,1$ e 0,5. O escore médio em cada caso indica a adequação da adaptação do setor. Isto é, em relação ao setor $A-R$, escores médios entre 2,61 e 3,0 indicam adaptação adequada; entre 2,00 e 2,60 pouco-adequada e entre 1 e 1,99, pouquíssimoadequada. Em relação ao setor $\mathrm{Pr}$, escores médios entre 1,61 e 2,00 indicam adaptação adequada; entre 1,00 e 1,60, pouco-adequada e entre 0 e 0,99, pouquíssimo-adequada. Para a classificação diagnóstica geral, os escores $(x)$ de cada grupo de eficácia da adaptação são os seguintes: Gr1 - Adaptação Eficaz (A-R e Pr adequados), $x \geq 4,22 ;$ Gr2 - Adaptação Ineficaz Leve (um setor adequado e outro pouco-adequado), 3,61 $x<4,22$; Gr3 - Adaptação Ineficaz Moderada (os dois setores com adaptação pouco-adequada, ou um é pouquíssimo- adequado e o outro adequado), 2,61 $\leq x<3,61$; Gr4 - Adaptação Ineficaz Severa (um setor pouco-adequado e o outro pouquíssimo-adequado), $2,00 \leq x<2,61$ e Gr5 Adaptação Ineficaz Grave (ambos pouquíssimoadequados), $1 \leq x<2,00$.

\section{Coping Response Inventory - Adult Form (CRI-A) (Moos, 1993)}

Instrumento de autorrelato desenvolvido para avaliar as estratégias de coping de acordo com o enfoque teórico transacional, segundo o qual o problema vivido, o enfrentamento e as consequências resultantes mantêm interação entre si. O teste avalia quatro estratégias de Aproximação ao problema e quatro estratégias de Evitação, sendo que em cada uma as duas primeiras são de tipo 
cognitivo e as duas últimas, de tipo comportamental. Cada uma das oito escalas de estratégias específicas está formada por seis itens. Os participantes devem responder a estes itens de acordo com uma escala Likert de quatro pontos em que 0 significa não ou nunca, 1 significa uma ou duas vezes, 2 significa bastante vezes e 3 significa sim, sempre. Além disso, o teste solicita que o sujeito descreva o acontecimento ou problema mais difícil que teve que resolver nos últimos doze meses e, em seguida, avalie 10 características do problema (experiência prévia, tempo de preparação, locus de controle, vivência do problema como um prejuízo ou um desafio, benefícios da vivência do problema etc.).

\section{Procedimentos}

A aplicação da EDAO-AR e da CRI-A ocorreu após a aprovação do projeto pelo Comitê de Ética da Universidade Federal de Goiás (Protocolo 274/11). Os dois instrumentos foram administrados no mesmo dia e de forma coletiva, em sala de aula dos participantes, e antecedidos da assinatura do Termo de Consentimento Livre e Esclarecido, para os maiores de 18 anos. Para os menores de idade, solicitou-se previamente o consentimento formal dos pais ou responsável legal.

Análise de dados. Os resultados foram analisados do ponto de vista descritivo, por meio de estatísticas de tendência central e dispersão (Média, Desvio padrão, Mínimo e Máximo). Empregou-se o Teste- $t$ para amostras independentes a fim de verificar a presença de diferenças nos escores da EDAOAR e CRI-A apresentados por homens e mulheres e, em seguida, correlação de Pearson para a avaliação do grau de associação entre os resultados da EDAO-AR e do CRI-A, assim como a associação entre idade dos participantes e os escores nos respectivos instrumentos. Destaca-se que as escolhas de técnicas paramétricas de correlação e comparação de médias foram sustentadas pela verificação de indicadores de normalidade das variáveis analisadas, valores de assimetria (Skewness), e valores de achatamento (Kurtosis) que se alocavam no intervalo entre -2 e 2 (George \& Mallery, 2010). As análises foram realizadas com apoio do pacote estatístico SPSS versão 22, adotando-se níveis de significância $p \leq 0,05$.

\section{RESULTADOS}

A Tabela 1 apresenta as médias, desvios padrão, valores mínimos e máximos nas escalas A-R, $\operatorname{Pr}$ e na EDAO-AR total. Apresenta, ainda, essas medidas para cada uma das oito escalas específicas avaliadas com o CRI-A, além das escalas globais Estratégias de Aproximação e Evitação do problema. Descrição dos resultados. 
Tabela 1

Escore médio, desvio padrão, valor mínimo e máximo da Escala Diagnóstica Adaptativa Operacionalizada de Autorrelato/EDAO-AR e do Coping Response Inventory- Adult Form/CRI-A ( $n=105)$.

\begin{tabular}{lcccc}
\hline \multicolumn{1}{c}{ ESCALA } & Média & DP & Mín. & Max. \\
\hline EDAO-AR & & & & \\
Afetivo-Relacional & 2,56 & 0,24 & 1,66 & 3 \\
Produtividade & 1,49 & 0,21 & 1,00 & 1,95 \\
Escore total & 4,04 & 0,41 & 2,79 & 4,86
\end{tabular}

\section{CRI-A}

$\begin{array}{lcccc}\text { Análise lógica } & 11,15 & 4,44 & 0 & 18 \\ \text { Reavaliação positiva } & 10,23 & 3,85 & 1 & 18 \\ \text { Busca de apoio } & 9,00 & 3,68 & 1 & 18 \\ \text { Resolução de problemas } & 11,21 & 4,19 & 0 & 18 \\ \text { Evitação cognitiva } & 9,70 & 4,18 & 0 & 18 \\ \text { Aceitação/Resignação } & 8,13 & 3,81 & 0 & 18 \\ \text { Busca de gratificação } & 8,50 & 4,61 & 0 & 18 \\ \text { alternativa } & 7,74 & 3,50 & 1 & 18 \\ \text { Descarga emocional } & 41,91 & 11,60 & 7 & 72 \\ \text { Estratégias de aproximação* } & 34,94 & 11,16 & 7 & 60 \\ \text { Estratégias de evitação** } & \end{array}$

Nota. *Estratégias de Aproximação = somatória dos escores das subescalas Análise lógica, Reavaliação positiva, Busca de apoio e Resolução de problemas. ** Estratégias de Evitação = somatória dos escores das subescalas: Evitação cognitiva, Aceitação ou Resignação, Busca de gratificação Alternativa, e Descarga emocional.

Em relação à eficácia adaptativa, os resultados médios denunciam pouca adequação no setor A-R e pouquíssima adequação no setor Pr para os sujeitos avaliados (Tabela 1). O valor médio do escore total indica Eficácia Adaptativa classificada como Ineficaz Leve. Para a CRI, observa-se que maiores médias são apresentadas para os fatores Resolução de problemas, Análise Lógica, e Reavaliação positiva, que dizem respeito a estratégias de Aproximação, que notadamente trata-se da estratégia global com mair valor médio. Por fim as estatítiscas mínima e máxima das variáveis analisadas indicam a variabilidade nas respostas dos participantes em ambas as escalas.

Comparando o desempenho na EDAO-AR e no CRI-A segundo o sexo dos participantes, não foram observadas diferenças significativas entre as médias de mulheres e homens para o setor A-R $(t(100)=-0,83, p=, 934$ e $M=2,55$ e 2,56 para mulheres e homens, respectivamente), para o setor $\operatorname{Pr}(t(99)=-741, p=$ 
$0,460, M=1,48$ e 1,52$)$, ou para os escores totais $(t(98)=-3,73, p=0,710$, $M=4,04$ e 4,08). Tampouco houve diferenças entre mulheres e homens nas estratégias de coping de Aproximação $(t(98)=-0,442, p=0,660, M=41,66$ e 43,07, para mulheres e homens respectivamente), e nas estratégias de coping de Evitação $(t(96)=0,988, p=0,329, M=41,66$ e 43,07) ou para a maioria das estratégias específicas de coping. Apenas para a estratégia de Descarga emocional foram observadas pontuações significativamente mais altas $(t(97)=0,930, p=0,02$, tamanho de efeito de $d=0,2)$ para as mulheres $(M=8,02)$ quando comparadas aos homens $(M=5,84)$.

Os coeficientes de correlação de Pearson entre as subescalas EDAO-AR (A$\mathrm{R}$; $\operatorname{Pr}$ e Escala total) e as subescalas que compõem o CRI-A, bem como entre idade dos participantes e as respectivas escalas são apresentados na sequência (Tabela 2).

Tabela 2

Correlação Pearson entre os escores da EDAO-AR, CRI-A (estratégias diretas e estratégias globais) e idade $(n=105)$.

\begin{tabular}{|c|c|c|c|c|}
\hline \multirow[b]{2}{*}{ CRI-A } & \multicolumn{4}{|c|}{ EDAO-AR } \\
\hline & $\begin{array}{c}\text { Afetivo- } \\
\text { Relacional }\end{array}$ & Produtividade & $\begin{array}{l}\text { Escore } \\
\text { Total }\end{array}$ & Idade \\
\hline Análise lógica & 0,001 & 0,006 & $-0,010$ & 0,067 \\
\hline Reavaliação positiva; & 0,074 & 0,100 & 0,093 & $0,326 * *$ \\
\hline Busca de apoio & 0,082 & 0,018 & 0,055 & $0,326 * *$ \\
\hline Resolução de problemas & $0,198^{*}$ & 0,115 & 0,138 & $0,215^{*}$ \\
\hline Evitação cognitiva & $-0,125$ & $-0,226^{*}$ & $-0,170$ & $-0,153$ \\
\hline Aceitação/Resignação & $-0,202^{*}$ & $-0,264 * *$ & $-0,257 * *$ & $-0,084$ \\
\hline $\begin{array}{l}\text { Busca de gratificação } \\
\text { alternativa }\end{array}$ & 0,034 & $-0,027$ & 0,008 & $0,238^{*}$ \\
\hline Descarga emocional & $-0,313^{* *}$ & $-0,311^{* *}$ & $-0,343 * *$ & 0,050 \\
\hline $\begin{array}{l}\text { Estratégias de } \\
\text { aproximação }\end{array}$ & 0,101 & 0,059 & 0,087 & $0,267 * *$ \\
\hline Estratégias de evitação & $-0,154$ & $-0,247^{*}$ & $-0,218^{*}$ & 0,038 \\
\hline \multicolumn{5}{|l|}{ EDAO-AR } \\
\hline Afetivo-Relacional & & & & 0,140 \\
\hline Produtividade & & & & 0,139 \\
\hline Escore Total & & & & 0,140 \\
\hline
\end{tabular}

Nota. $* \mathrm{p}<0,05, * * \mathrm{p}<0,01$.

Pela Tabela 2, pode-se verificar correlações significativas de baixas magnitudes entre os escores do setor A-R e a estratégia de Resolução de problema. Além disso, os escores deste setor correlacionaram-se negativa e moderadamente com estratégias diretas de Aceitação/Resignação e Descarga emocional, indicando que aqueles indivíduos que enfrentam diretamente seu 
problema buscando resolvê-lo com certo controle emocional, tendem a dar respostas mais adequadas para a solução de seus problemas.

O setor $\operatorname{Pr}$ da EDAO-AR apresentou correlações negativas significativas de baixa magnitude com as estratégias de evitação avaliadas pelo CRI-A: Evitação cognitiva, Aceitação/Resignação, Descarga emocional, e consequentemente nas Estratégias Globais de Evitação, indicando que aqueles indivíduos que evitam cognitivamente seus problemas tendem a dar respostas pouco ou pouquíssimo adequadas para a solução de seus problemas.

Os escores totais da escala EDAO-AR apresentavam correlações significativa negatigativas e de baixa magnitude com as estratégias de Aceitação/Resignação e Descarga emocional, bem como com a estratégia gobal Evitação. Esses resultados indicaram a tendencia de que quanto maior o uso desse tipo de estratégia, pior é eficácia adaptativa.

Por fim, observa-se na Tabela 2 índices de correlação significativos positivos de baixa magnitude entre idade dos participantes e as estratégias de Reavaliação positiva, Busca de apoio, Resolução de problemas, Busca de gratificação alternativa e consequentemetne com Estratégias global de aproximação, tais resultados indicaram que com o advento da idade as pessoas tendem a fazer uso estratégia mais adaptativas no enfrentamento de situações estressantes.

\section{DISCUSSÃO}

Este estudo objetivou buscar relações conceituais e empíricas entre eficácia adaptativa e coping. Foram analisados os dados descritivos de dois instrumentos de medida, um de eficácia adaptativa, a EDAO-AR, e outro de coping, o CRI-A. São consideradas respostas pouco adequadas aquelas que solucionam o problema, trazem satisfação ao sujeito, mas criam conflito interno, ou ainda com o ambiente. Outra possibilidade de adaptação pouco-adequada é ensejada pela resposta que soluciona o problema, contudo não traz prazer ao sujeito, ainda que não cause conflito interno ou externo (Simon, 1983/1989, 2005). É portanto preocupante verificar que, tanto no âmbito das relações interpessoais quanto da produtividade, o padrão médio de respostas dos univeritários de instituição pública do centro-oeste corresponda aos de soluções pouco-adequadas. Por outro lado, em relação à configuração da adaptação (Tabela 1), tanto para os homens quanto para as mulheres, foi em média Ineficaz Leve (Gr2), o que corresponde à adaptação adequada em um dos setores e pouco-adequada em outro. Ou seja, a tendência foi a de os participantes apresentarem adaptação pouco adequada apenas em um dos setores. Do ponto de vista do funcionamento geral, este se traduz pela presença de "sintomas neuróticos brandos, ligeiros traços caracterológicos, algumas 
inibições" (Simon, 1997, p.92), o que não chega a caracterizar um quadro de patologia grave, conforme se espera de estudantes universitários.

As pontuações médias nas escalas de coping apresentadas pelos participantes monstraram-se superiores àquelas descritas em estudos internacionais, como diante de população espanhola (Kirchner \& col., 2008) e das médias descritas no manual do autor (Moos, 1993). Mais especificamente, são as estratégias do grupo de Evitação ao problema aquelas que apresentam níveis mais elevados, que os descritos na literatura internacional. Este aspecto poderia indicar uma característica cultural, como maior uso de um conjunto de estratégias de coping por parte dos estudantes brasileiros estudados como forma de enfrentamento a seus problemas. Além disso, pode indicar um enfrentamento menos adaptado, uma vez que o maior uso concentra-se especialmente entre as estratégias do tipo evitação do problema, que são descritas como relacionadas a menores índices de saúde e maiores índices de psicopatologia (Moos, 1993).

A ausência de diferenças entre os participantes do sexo masculino e feminino, no que respeita à qualidade da adaptação no setor $A-R$, da $\operatorname{Pr} e$ também em relação ao escore total da EDAO-AR, sugere que tanto os universitários quanto as universitárias estariam respondendo de forma semelhante às contingências afetivo-relacionais e também em relação às situações e desafios do meio escolar. Nos dias atuais, não há, de fato, motivos para supor que a qualidade da adaptação estaria ligada ao sexo. Em relação ao uso das estratégias de coping por estudantes universitários, os escores médios observados neste estudo encontram-se em consonância com aqueles descritos por Tomaz e Zanini (2009). Vale ressaltar todavia, que nos estudos internacionais, quando comparadas as médias obtidas entre mulheres e homens, elas apresentam uso significativamente maior de estratégias de coping, diferente dos resultados encontrados nesta pesquisa, onde os grupos apresetaram escores médios semelhantes, exceto no caso do uso da estratégia de Descarga emocional, o que parece ser um consenso na literatura tanto brasileira como internacional, que mulheres tendem a utilizar significativamente mais estratégias do tipo Descarga emocional do que homens (Kirchner et al., 2008; Moos, 1993; Paula Jr. \& Zanini, 2011; Tomaz \& Zanini, 2009). Contudo sugere-se cautela na generalização dos resultados obtidos, uma vez que amostra foi composta maioritariamente por mulheres, neste direção novos estudos devem contar com amostras balanceadas para verificação da reprotudibilidade destes resultados.

Em relação à hipótese de que há sobreposição entre os dois construtos avaliados pela EDAO-AR e CRI-A, ela foi parcialmente corroborada pelos resultados encontrados (Tabela 2 ). Observa-se que as correlações variaram entre 0,00 e 0,20 para as estratégias de Aproximação ( $A L, R P, B A$ e $R P$ ) e Estratégia Global de aproximação (Aprox.) e a qualidade da adaptação nas escalas A-R e Pr e EDAO-AR total. Isto é, as correlações tenderam a ser positivas e baixas, ou inexistentes tanto para as escalas $A-R$ e $\operatorname{Pr}$ quanto em relação à 
medida de eficácia da adaptação (EDAO-AR total). Apenas em relação à estratégia de coping Resolução de problemas (RP) e a escala $A-R$, a correlação chegou a ser significativa $(p<0,05)$. Conforme referido, a RP corresponde a tentativas comportamentais de ações diretas para solução do problema. Nesse sentido, os dados sugerem que em situações de estresse, quanto melhor a adequação da adaptação no setor $A-R$, maior a tendência a se recorrer a esse tipo de estratégia para enfrentá-las. Em relação às demais estratégias de Aproximação, elas não guardam associação com a adequação da adaptação, sugerindo tratarem-se de características cognitivas (Análise lógica e Reavaliação positiva) ou comportamentais (Busca de apoio), independentes da natureza da situação (Afetivo-Relacional ou Produtividade).

De forma geral, os resultados mostraram associação negativa e moderada entre as estratégias de Evitação ( $E C, A R, B G A$ e $D E$ ) e Estratégia Global de Evitação (Evit.), medidas pelo CRI-A e a qualidade da adaptação no setor A-R, Pr e Configuração Adaptativa Total, de acordo com a EDAO-AR (Tabela 2). As correlações foram negativas e moderadas $(p<0,05)$, especialmente entre as estratégias de Aceitação/Resignação (AR) e Descarga emocional (DE) e as escalas da EDAO-AR (A-R, $\operatorname{Pr}$ e total). As estratégias cognitivas de aceitação ou resignação (AR) referem-se a tentativas de aceitação do problema ou adoção de atitudes de resignação frente a ele. $\mathrm{E}$ as estratégias incluídas na categoria Descarga emocional (DE) correpondem a tentativas comportamentais de reduzir a tensão decorrente de um problema, por meio da expressão de sentimentos negativos. Conforme apontaram os resultados, elas tendem a diminuir com a melhora da adequação da adaptação.

Quanto à estratégia de Busca da Gratificação Alternativa (BGA), os resultados apontaram ausência de associação (correlações próximas de zero) com a qualidade da adaptação, tanto na esfera dos relacionamentos interpessoais, quando da produtividade, o que, por sua vez se reflete na configuração da adaptação (Tabela 2). A estratégia de BGA leva o indivíduo a envolver-se em atividades substitutivas que gerem satisfação e evitem o malestar causado pela vivência do problema. Ou seja, trata-se de respostas comportamentais frente à situação estressante, que eventualmente geram alguma satisfação, mas não resolvem o problema. Do ponto de vista da adaptação, essa estratégia corresponderia à adaptação pouco-adequada.

Quanto aos índices de correlação positivos e moderados observados entre idade e as estratégias Reavaliação positiva, Busca de apoio, Resolução de problema, Busca de gratificação alternativa e, por conseguinte, Estratégias de aproximação, indicam que as pessoas tendem a fazer uso de estratégias mais realistas e adaptadas com o passar da idade.

De modo geral, observaram-se mais associações entre a EDAO-AR e estratégias de coping de Evitação do que entre as estratégias de coping de Aproximação. Embora teoricamente isto represente um paradoxo, o uso de 
coping associa-se negativamente a medidas de saúde, ou seja, são os enfrentamentos ineficazes aqueles que se associam (negativamente) com medidas de saúde, mas não os eficazes. Este tipo de contradição teórica está em consonância com o observado em estudos internacionais (Aldwin \& Revenson, 1997).

No que respeita às correlações entre a eficácia adaptativa e as estratégias de coping, as associações negativas entre a adequação do Setor A-R e as estratégias Aceitação/Resignação e Descarga Emocional, podem indicar que estas, quando usadas, tenderiam a não trazer satisfação e/ou a gerar conflito. Da mesma maneira ocorreria no Setor $\operatorname{Pr}$ em relação às estratégias de Evitação Cognitiva, Aceitação/Resignação, Descarga Emocional e Evitação. Dentre estas estratégias, particularmente Evitação Cognitiva, Evitação e Aceitação/Resignação mostraram-se preditivas de níveis mais baixos de satisfação com a vida em estudo conduzido por Pretorius, Walker e Esterhuyse (2010).

Por outro lado, as correlações moderadas ou ainda, as inexistentes, sugerem que o escopo de respostas comportamentais medido pelos itens do CRIA não se sobrepõe às respostas previstas na EDAO-AR. Isto é, os instrumentos mediriam estratos comportamentais específicos e diferentes, resultando na ausência de correlação entre as medidas que eles permitem. Um estudo da validade de conteúdo em que ambos os instrumentos venham a ser comparados, poderá eventualmente, dirimir essa questão.

\section{CONSIDERAÇÕES FINAIS}

Ao se submeter duas medidas de avaliação, uma de eficácia adaptativa e outra de coping à investigação de possíveis relações empíricas entre os dois construtos, as correlações significativas obtidas foram de baixa magnitude, positivas ou negativas, indicando pouca superposição entre eles. Quando se atenta para a definição teórica de ambos os conceitos, verifica-se que o coping está associado especificamente a situações de estresse, que demandam algum tipo de enfrentamento, enquanto que o conceito de eficácia adaptativa se aplica a toda e qualquer resposta, que tenha como finalidade a resolução de uma situação-problema, independentemente de esta ser considerada estressante pelo indivíduo. Nesse sentido, as respostas (cognitivas e comportamentais) de coping podem ser vistas como parte das respostas adaptativas. Ademais, o construto eficácia adaptativa ao prever a qualificação da resposta considerando-a adequada, pouco-adequada ou pouquíssimo-adequada, avança no sentido de qualificar a resposta escolhida, ou o tipo de estratégia de enfrentamento escolhido em função da satisfação que o indivíduo sente ao tentar resolver um problema e da resultante ausência de conflito (interno e ou com o ambiente) (Simon, 1983; 1989). 
Essa afirmação aplica-se especialmente às estratégias de Evitação no enfrentamento de situações estressantes. Nesses casos, a qualidade adaptativa das respostas tende a ser inversamente proporcional à utilização das estratégias cognitivas e comportamentais, mas de forma apenas moderada e não aplicável a todas às modalidades de estratégia de coping.

Quanto às estratégias de Aproximação, não há evidências de que estejam associadas à qualidade da adaptação. Mesmo em relação à estratégia Resolução de problemas, que nesse estudo correlacionou-se positivamente com a qualidade da adaptação da escala A-R, pode ter se tratado de uma característica específica dessa amostra. Nesse sentido, novas investigações, com estratos mais heterogêneos de universitários, mostram-se necessárias. Para tanto, deve-se procurar amostras compostas por universitários de diferentes cursos superiores, de diferentes regiões do país e provenientes de instituições públicas e privadas. Pequenas diferenças na composição de amostras podem, de fato, levar a resultados divergentes, ou ainda, a erros de medidas (precisão), intrínsecos a avaliações propiciadas por qualquer instrumento empiricamente desenvolvido.

Resumindo, pode-se dizer que apesar de guardarem entre si algum grau de associação e de sobreposição, os construtos de eficácia adaptativa e coping abordam aspectos diferentes das atividades humanas. Nessa medida, o emprego concomitante de avaliações de ambos os construtos pode ser útil na prática profissional de psicólogos e também em pesquisas, fornecendo avaliações de aspectos complementares do funcionamento geral de universitários e provavelmente de adultos jovens, de uma maneira geral.

Por fim, destacam-se alguns dos limites da presente pesquisa que se baseia em uma amostra composta por conveniência, oriunda de uma região específica do país, Centro-Oeste. Em pesquisas futuras esforços devem ser realizados no sentido de envolver amostras mais heterogêneas e representativas que possibilitem a avaliação de outras características dos estratos amostrais, por exemplo, a comparação dos escores em função de diferentes faixas etárias. O que possibilitaria corroborar ou refutar as hipóteses levantadas na presente pesquisa.

\section{REFERÊNCIAS}

Aguilar-Vafaie, M. E., \& Abiari, M. (2007). Coping Response Inventory: Assessing coping among Iranian college students and introductory development of an adapted Iranian Coping Response Inventory (CRI). Mental Health, Religion \& Culture, 10(5), 489-513.

Aldwin, C. (1994). Stress, coping and development: An integrative perspective. New York London: The Guilford Press. 
Aldwin, C., \& Revenson, T. (1997). Does coping help? A re-examination of the relation between coping and mental health. Journal of Personality and Social Psychology, 53, 337-348.

Folkman, S. (Ed.) (2011). The Oxford handbook of stress, health, and coping. New York: Oxford University Press.

Folkman, S., \& Lazarus, R. S. (1980). An analysis of coping in a middle-aged community sample. Journal of Health and Social Behavior, 21, 219-239.

Kirchner, T., Forns, M., Muñoz, D., \& Pereda, N. (2008). Psychometric properties and dimensional structure of the Spanish version of the Coping Responses Inventory - Adult Form. Psicothema, 20(4), 902-909.

Kohli, S., Batra, P., \& Aggarwal, H., K. (2011). Anxiety, locus of control, and coping strategies among end-stage renal disease patients undergoing maintenance hemodialysis. Indian Journal of Nephrology, 21(3), 177-181.

Laloni, D. T. (2001). Escala de Avaliação de Sintomas-90-R-SCL-90-R: adaptação, precisão e validade. Tese de Doutorado, Pontifícia Universidade Católica de Campinas. Disponível em: http://www.bibliotecadigital.puccampinas.edu.br/tde_busca/arquivo.php?codArquivo $=294$

Lazarus, R. S., \& Folkman, S. (1984). Stress, appraisal, and coping. New York, NY: Springer, 1984.

Moos, R. H. (1993). Coping Response Inventory Youth Form: Professional manual. Odessa, FL: Psychological Assessment Resources.

Norcross, J. C. (2011). Psychotherapy relationships that work: Evidence-Based Responsiveness ( $2^{\mathrm{a}}$ ed.) New York, NY: Oxford University Press.

Paula Junior, W., \& Zanini, D. S. (2011). Estratégias de coping de pacientes oncológicos em tratamento radioterápico. Psicologia: Teoria e Pesquisa, 27(4), 491-497. doi: 10.1590/S0102-37722011000400013

Pretorius, C., Walker, S.P. \& Esterhuyse, K.G.F. (2010). Coping responses as predictors of satisfaction with life amongst a group of patients diagnosed with diabetes mellitus, Health SA Gesondheid, 15(1). doi: 10.4102/hsag.v15i1.513

Santeiro, F. R. M., Zanini, D. S., \& Santeiro, T. V. S. (2013). Análise empírica de tendências na produção científica sobre coping (SciELO, 1993/2012) (manuscrito submetido).

Simon, R. (1989). Psicologia clínica preventiva: Novos fundamentos. São Paulo: EPU. (Original publicado em 1983: Vetor)

Simon, R. (1997). Proposta de redefinição da Escala Diagnóstica Adaptativa Operacionalizada. Boletim de Psicologia, 47(107), 85-93.

Simon, R. (2005). Psicoterapia breve operacionalizada: Teoria e técnica. São Paulo: Casa do Psicólogo.

Schinke, R.J., Battochio, R.C., Lidor, R., Tenenbaum, G., Dube, T.V., \& Lane, A.M. (2012). Adaptation processes affecting performance in elite sport. Journal of Clinical Sport Psychology, 6, 180-195. 
Schinke, R.J., Tenenbaum, G., Lidor, R., \& Battochio, R.C. (2010). Adaptation in action: the transition from research to intervention. The Sport Psychologist, 24, 542-557.

Vaillant, G.E. (2002). Aging well. Boston: Little, Brown \& Company.

Yoshida, E. M. P. (2012). Escala Diagnóstica Adaptativa Operacionalizada de Auto-Relato (EDAO-AR) ( $2^{a}$ versão). Pontifícia Universidade Católica de Campinas (manuscrito).

Yoshida, E. M. P. (2013). Escala Diagnóstica Adaptativa Operacionalizada de Autorrelato - EDAO-AR: Evidências de validade. Paidéia, 23(54), 83-91. doi: $10.1590 / 1982-43272354201310$

Yoshida, E. M. P., Enéas, M. L. E., \& Santeiro, T. V. (2010). Escala Diagnóstica Adaptativa Operacionalizada (EDAO): Avaliação da qualidade da eficácia adaptativa. In A. A. A. Santos, F. F. Sisto, E. Boruchovitch \& E. Nascimento (Eds.), Perspectivas em avaliação psicológica (pp. 211-228). São Paulo: Casa do Psicólogo.

Zanini, D. S., Forns, M., \& Kirchner, T. (2005). Coping response and problem appraisal in Spanish adolescent. Perceptual and Motor Skills, 100, 153-166.

Zanini, D., \& Queiroz, I. P. (2011). Apoio social, coping e suas repercussões no humor depressivo. Estudos, 38(1/3), 133-149.

Zanini, D.S., Mendonça, Forns, M., \& Kirchner, T. (2010). Psychometric properties of the Coping Response Inventory with brazilian adolescentes. Psychological Reports, 107, 617-628. doi: 10.2466/08.17.20.PR0.107.5.617-628

Sobre os autores

Tales Vilela Santeiro é psicólogo pela Universidade de Franca, mestre e doutor em Psicologia pela Pontifícia Universidade Católica de Campinas. É Professor do Departamento de Psicologia da Universidade Federal do Triângulo Mineiro. talessanteiro@hotmail.com

Elisa Medici Pizao Yoshida é doutora em Psicologia, docente do programa de pósgraduação stricto sensu em Psicologia da PUC-Campinas entre 1990 e 2013. eyoshida.tln@terra.com.br

Evandro Morais Peixoto é psicólogo Graduado em Psicologia pela Universidade Presbiteriana Mackenzie (2010). Mestre em Psicologia como Profissão e Ciência, com ênfase em avaliação psicológica, pela Pontifícia Universidade Católica de Campinas (2012). Doutorando em Psicologia pela mesma universidade com estágio doutoral desenvolvido na Université du Québec à Trois-Rivières - QC Canadá. epeixoto_6@hotmail.com

Glaucia Mitsuko Ataka da Rocha é pós-doutoranda no Departamento de Psicologia Clínica da Universidade de São Paulo, doutora em Psicologia como Profissão e Ciência e Mestre em Psicologia Clínica pela Pontifícia Universidade de São Paulo.gmarocha@gmail.com

Daniela Sacramento Zanini é psicóloga pela Pontifícia Universidade Católica de Goiás, doutora em Psicologia Clínica e Saúde pela Universidade de Barcelona. É professora do Programa de Pós Graduação Mestrado e Doutorado em Psicologia e 
Departamento de Psicologia pela Pontifícia Universidade Católica de Goiás e bolsista produtividade pelo CNPq. dazanini@yahoo.com.

Recebido em: 16/12/2015 $1^{\text {a }}$ revisão em: 04/02/2016

$2^{a}$ revisão em: 27/02/2016 Aceito em: 08/03/2016 